\title{
UNIQUENESS OF MINIMISERS FOR A GRÖTZSCH-BELINSKII TYPE INEQUALITY IN THE HEISENBERG GROUP
}

\author{
ZOLTÁN M. BALOGH, KATRIN FÄSSLER, AND IOANNIS D. PLATIS
}

\begin{abstract}
The modulus method introduced by H. Grötzsch yields bounds for a mean distortion functional of quasiconformal maps between two annuli mapping the respective boundary components onto each other. P. P. Belinskiu studied these inequalities in the plane and identified the family of all minimisers. Beyond the Euclidean framework, a Grötzsch-Belinskiu-type inequality has been previously considered for quasiconformal maps between annuli in the Heisenberg group whose boundaries are Korányi spheres. In this note we show that - in contrast to the planar situation - the minimiser in this setting is essentially unique.
\end{abstract}

\section{INTROdUCtion AND STATEMENT OF RESUlts}

Ever since the pioneering work by H. Grötzsch and the groundbreaking treatise by L. Ahlfors, geometric methods have played an important role in the theory of quasiconformal maps and they have been crucial for the development of this theory in abstract metric measure spaces of controlled geometry. Of particular importance is the modulus of curve families, which yields bounds for the distortion of maps subject to certain boundary conditions. In specific situations, such modulus-distortion estimates allow us to identify extremal quasiconformal maps, that is, minimisers for a certain distortion functional. However, the modulus method does not tell in general whether the minimiser is unique. Questions of uniqueness require typically also analytic techniques and have therefore, to our knowledge, not been studied much outside the Riemannian framework. In this paper, we address a uniqueness question in the setting of the sub-Riemannian Heisenberg group. The problems we are dealing with are motivated by the following result of Belinskiǔ, [7, Lemma 1], which we restate here as follows:

Theorem 1.1. Fix $k>0$ and consider two annuli in the complex plane,

$$
A_{a, b}=\{z \in \mathbb{C}: a<|z|<b\} \quad \text { and } \quad A_{a^{k}, b^{k}}=\left\{z \in \mathbb{C}: a^{k}<|z|<b^{k}\right\}
$$

Further, let $\mathcal{F}$ be the family of quasiconformal maps $f: A_{a, b} \rightarrow A_{a^{k}, b^{k}}$ which map boundary components to respective boundary components. For each $f \in \mathcal{F}$ with dilatation $K_{f}$, the following inequality holds:

$$
k^{-1} \leq \frac{\int_{A_{a, b}} \frac{K_{f}(z)}{|z|^{2}} d \mathcal{L}^{2}(z)}{2 \pi \log \left(\frac{b}{a}\right)} .
$$

Received by the editors November 7, 2014 and, in revised form, March 31, 2015.

2010 Mathematics Subject Classification. Primary 30L10, 30C75.

Key words and phrases. Heisenberg group, extremal quasiconformal mappings, modulus method, mean distortion, uniqueness of minimisers.

This research was supported by the Swiss National Science Foundaton. 
The inequality is strict if $k>1$. In the case $0<k<1$, equality is attained by the quasiconformal maps $f$ of the form $f(z)=|z|^{k} e^{i \Psi(\arg z)}$ for a real-valued function $\Psi$. Moreover, when $k \in(0,1)$, up to rotations around the origin, the map $f(z)=z|z|^{k-1}$ is the only (orientation preserving) map of constant distortion in $\mathcal{F}$ that realises the above inequality as an equality. It is also of least possible maximal distortion in $\mathcal{F}$.

Remark 1.2. The condition that $f(z)=|z|^{k} e^{i \Psi(\arg z)}$ ought to be a quasiconformal map imposes restrictions on $\Psi$. For smooth maps, one can see by a simple computation that $\Psi$ has to be a diffeomorphism between intervals of length $2 \pi$ with the property that $\left|\Psi^{\prime}\right| \geq k$. Note further that while Belinskil carries out the proof for continuously differentiable maps, he mentions that the arguments remain true under the assumption that the map has only weak derivatives that belong to $L^{2}$.

Our goal is to generalise Belinskiǔ's result to the Heisenberg group. The Heisenberg group $\mathfrak{H}$ is a Lie group modeled on $\mathbb{R}^{3}$ or $\mathbb{C} \times \mathbb{R}$ endowed with the noncommutative group law

$$
(z, t) *\left(z^{\prime}, t^{\prime}\right)=\left(z+z^{\prime}, t+t^{\prime}+2 \Im\left(z \bar{z}^{\prime}\right)\right), \quad(z, t),\left(z^{\prime}, t^{\prime}\right) \in \mathbb{C} \times \mathbb{R},
$$

and the metric

$$
d_{H}(p, q)=\left\|q^{-1} * p\right\|_{H}, \quad \text { where }\|(z, t)\|_{H}=\sqrt[4]{|z|^{4}+t^{2}} .
$$

Balls centred at $p$ of radius $r$ with respect to the metric $d_{H}$ will be denoted by $B(p, r)$.

The Heisenberg group can be equipped with a contact form $\tau$, the kernel of which is spanned by the real and imaginary parts of the horizontal vector fields $Z=\partial_{z}+\mathrm{i} \bar{z} \partial_{t}$ and $\bar{Z}=\partial_{\bar{z}}-\mathrm{i} z \partial_{t}$. Quasiconformal maps in this setting were first studied by Mostow, Pansu, Korányi and Reimann [12, 13, 15, 16], and they can be defined in a metric sense as homeomorphisms between domains in $\mathfrak{H}$ such that

$$
\limsup _{r \rightarrow 0+} \frac{\sup _{d_{H}(p, q)=r} d_{H}(f(p), f(q))}{\inf _{d_{H}(p, q)=r} d_{H}(f(p), f(q))} \leq H<\infty, \quad \text { for all } p .
$$

Korányi and Reimann established an equivalent analytic characterization of quasiconformal maps. A homeomorphism $f=\left(f_{1}+\mathrm{i} f_{2}, f_{3}\right)=\left(f_{I}, f_{3}\right)$ is (orientation preserving) quasiconformal if it belongs to the horizontal Sobolev space $H W_{l o c}^{1,4}$ which ensures that its components have weak $Z$ and $\bar{Z}$ derivatives - and if it satisfies the distortion condition

$$
K_{f}:=\frac{\left|Z f_{I}\right|+\left|\bar{Z} f_{I}\right|}{\left|Z f_{I}\right|-\left|\bar{Z} f_{I}\right|} \leq K<\infty, \quad \text { almost everywhere }
$$

see [10,13]. Here, "orientation-preserving" is to be understood as in (2.7).

In this note we are concerned with uniqueness of minimisers for a functional that involves the distortion $K_{f}$ and is related to the conformal 4-modulus

$$
\operatorname{Mod}(\Gamma)=\inf _{\rho \in \operatorname{adm}(\Gamma)} \int_{\mathfrak{H}} \rho^{4} \mathrm{~d} \mathcal{L}^{3}
$$

of curve families $\Gamma$ in $\mathfrak{H}$. Here, $\operatorname{adm}(\Gamma)$ denotes the family of all Borel functions $\rho: \mathfrak{H} \rightarrow[0,+\infty]$ such that $\int_{\gamma} \rho \mathrm{d} s \geq 1$ for all locally rectifiable $\gamma$ in $\Gamma$. 
Definition 1.3. Let $f: \Omega \rightarrow \Omega^{\prime}$ be a quasiconformal map between domains of $\mathfrak{H}$ and let $\rho: \Omega \rightarrow[0, \infty]$ a Borel function. The mean distortion $\mathfrak{M}(f, \rho)$ is given by

$$
\mathfrak{M}(f, \rho)=\frac{\int_{\Omega} K_{f}^{2}(p) \rho^{4}(p) d \mathcal{L}^{3}(p)}{\int_{\Omega} \rho^{4}(p) d \mathcal{L}^{3}(p)} .
$$

Our focus lies on the minimisation of the functional $\mathfrak{M}(f, \rho)$ for $\rho$ equal to the extremal density $\rho_{0}$ of the 4 -modulus of curves connecting the boundary components of a spherical annulus. A minimiser for this extremal problem is the "radial stretch map", which can be best described using logarithmic coordinates on the Heisenberg group. Logarithmic coordinates [17] for $\mathfrak{H}$ are given by the map

$$
\Phi(\xi, \psi, \eta)=\left(\mathrm{i} \cos ^{1 / 2} \psi e^{\frac{\xi+\mathrm{i}(\psi-3 \eta)}{2}},-\sin \psi e^{\xi}\right) .
$$

The map $\Phi$ is surjective from $\mathbb{R} \times(-\pi / 2, \pi / 2) \times \mathbb{R}$ onto $\mathfrak{H}$ minus the vertical axis, and it becomes injective when $\eta$ is restricted to an appropriate interval of length $4 \pi / 3$.

In [4], we proved the following theorem for spherical Korányi annuli

$$
S_{a, b}=\left\{p \in \mathfrak{H}: a<\|p\|_{H}<b\right\} \quad \text { and } \quad S_{a^{k}, b^{k}}=\left\{p \in \mathfrak{H}: a^{k}<\|p\|_{H}<b^{k}\right\} .
$$

Theorem 1.4. For any $k \in(0,1)$, the stretch map $f_{k}$,

$$
f_{k}(\xi, \psi, \eta)=\left(k \xi, \tan ^{-1}\left(\frac{\tan \psi}{k}\right), \eta\right)=(\Xi, \Psi, H)
$$

is an orientation preserving quasiconformal map from the Heisenberg spherical ring $S_{a, b}$ onto the Heisenberg spherical ring $S_{a^{k}, b^{k}}$. For

$$
\rho_{0}(\xi, \psi, \eta)=(\log (b / a))^{-1} e^{-\xi / 2} \cos ^{1 / 2}(\psi) \mathcal{X}_{S_{a, b}}(\xi, \psi, \eta)
$$

and $\Omega=S_{a, b}$, it minimises the mean distortion $\mathfrak{M}\left(f, \rho_{0}\right)$ within the class $\mathcal{F}$ of quasiconformal maps $f: S_{a, b} \rightarrow S_{a^{k}, b^{k}}$ which map the boundary components of $S_{a, b}$ to the respective boundary components of $S_{a^{k}, b^{k}}$, so that for any such $f$ we have

$$
k^{-3}=\mathfrak{M}\left(f_{k}, \rho_{0}\right) \leq \mathfrak{M}\left(f, \rho_{0}\right),
$$

As we illustrate in [4, the Heisenberg stretch map has analogous properties as the radial stretch $f_{k}(z)=z|z|^{k-1}$ in the complex plane, but it differs in one important aspect: the distortion of the Heisenberg stretch is not constant. The maximal distortion is attained on the plane $t=0$, and it is larger than the bound provided by the modulus estimate for the connecting curves in the annulus. It is therefore possible that there exists a map in $\mathcal{F}$ with maximal distortion smaller than that of $f_{k}$. We already know from [4] that such a minimiser could not be sphere- and $t$-axis preserving. The main result of this paper shows that if a minimiser $f$ for the maximal distortion with $\left\|K_{f}\right\|_{\infty}<\left\|K_{f_{k}}\right\|_{\infty}$ exists, it cannot at the same time minimise the mean distortion functional.

In this paper, we consider the question of finding all minimisers in (1.3). Our main result states that, in strong contrast to the planar case, in the Heisenberg group, such minimisers are essentially unique. We wish to emphasise that the minimisers which Belinskiu found are more than those which one obtains trivially as compositions of the prototypical stretch map $f_{k}(z)=z|z|^{k-1}$ with conformal maps. In particular, the rigidity of conformal maps in the Heisenberg group alone is not sufficient to explain the lack of other minimisers. 
Theorem 1.5. For $k \in(0,1)$, let $\mathcal{F}$ be the family of orientation preserving quasiconformal maps $f: S_{a, b} \rightarrow S_{a^{k}, b^{k}}$ which extend homeomorphically to the boundary and map boundary components to respective boundary components. Then for each $f \in \mathcal{F}$, inequality (1.3) holds and equality is attained only by the stretch map $f_{k}$ given in (1.1) and by compositions of $f_{k}$ with rotations $m_{\alpha}(z, t)=\left(e^{\mathrm{i} \alpha} z, t\right)$.

The main reason for the uniqueness in Theorem 1.5] is to be found in the contact condition, i.e., it is due to the fact that quasiconformal maps in the Heisenberg group preserve the contact structure almost everywhere; see e.g., [10, 13, 15] (Mostow, Korányi-Reimann). The contact condition together with the minimising property imposes a strong rigidity expressed by an almost overdetermined system of first order PDEs whose only solution will eventually be the stretch map.

In Section 2, we prove Theorem 1.5 by gradually deriving information on the minimiser, which eventually leaves no other possibility for it than to be the stretch map or a composition of stretch and rotation. The structure of the proof is as follows:

In Section 2.1, we study the behavior of minimisers on the family of radial curves that are horizontally normal to Korányi spheres. If we also allow the vertical axis as a degenerate radial curve, we can show that minimisers map the family of radials in $S_{a, b}$ to the radials in $S_{a^{k}, b^{k}}$. Radial curves point in the direction of the least possible stretching of solutions to our minimisation problem. This minimial stretching property coupled with the contact property, the boundary conditions and the fact that minimisers essentially push forward the extremal density in $S_{a, b}$ to the extremal density in the target, provide information on the amount of stretching along radial curves. This information yields that minimisers are sphere-preserving as shown in Section 2.2. Each Korányi sphere is foliated by a family of horizontal curves, which we call spherical arcs. The family of all spherical arcs is in some sense dual to the radials, and we prove in Section 2.3 that it has to be preserved by minimisers as well. In particular, this forces such maps to fix the vertical axis. Maps with this property can be conveniently described in logarithmic coordinates, which are well adapted to the geometry of the problem. Maps that preserve radials and spherical arcs satisfy a functional equation, whose only solution are compositions of the radial stretch map with rotations, as discussed in Section 2.4. We believe that this proof strategy, which is exemplified here in the prototypical situation of radial stretching in $\mathfrak{H}$, could provide a scheme to follow also for other uniqueness questions in minimisation of mean distortion functionals. We conclude the main part of the paper with final remarks and open questions in Section 3. Several technical results are collected in an appendix at the end of the paper.

\section{Proof OF THE MAIN RESUlT}

2.1. Stretching along radials. This section is devoted to curves that are normal to Korányi spheres with respect to a product $\langle\cdot, \cdot\rangle_{0}$ associated to the horizontal structure, and to the effect of minimisers on such curves.

2.1.1. Radial curves. We are going to prove Theorem [1.5 by studying the behavior of minimisers along particular horizontal curves: the radial curves. A horizontal curve is an absolutely continuous map $\gamma:[a, b] \rightarrow \mathfrak{H}$ with the property that in almost every point $s \in[a, b]$ its tangent $\dot{\gamma}(s)$ belongs to the horizontal subspace $H_{\gamma(s)} \mathfrak{H}$ of $T_{\gamma(s)} \mathfrak{H}$ spanned by $X_{\gamma(s)}=2 \Re\left(Z_{\gamma(s)}\right)$ and $Y_{\gamma(s)}=-2 \Im\left(Z_{\gamma(s)}\right)$. For such 
$\gamma(s)=\left(\gamma_{1}(s), \gamma_{2}(s), \gamma_{3}(s)\right)$ we have that

$$
\dot{\gamma}(s)=\dot{\gamma}_{1}(s) X_{\gamma(s)}+\dot{\gamma}_{2}(s) Y_{\gamma(s)} .
$$

In the definition of radial curves, we use the notation $u=\|\cdot\|_{H}$. Here and in the following, we denote by $\nabla_{0} h$ the horizontal gradient $(X h) X+(Y h) Y$ of a function $h: \mathfrak{H} \rightarrow \mathbb{R}$. We write $\langle\cdot, \cdot\rangle_{0}$ for the inner product that makes $\{X, Y\}$ orthonormal, and $|\cdot|_{0}$ for the corresponding norm.

For $p \in \mathfrak{H} \backslash$ (t-axis), we consider the Cauchy problem

$$
\left\{\begin{array}{l}
\frac{\partial}{\partial s} \phi(s, p)=\frac{u(\phi(s, p))}{s} \frac{\nabla_{0} u(\phi(s, p))}{\left|\nabla_{0} u(\phi(s, p))\right|_{0}^{2}}, \\
\phi(1, p)=p .
\end{array}\right.
$$

The solutions for fixed $p$ are horizontal curves with tangent parallel to $\nabla_{0} u$.

Definition 2.1 (Radial curve). A horizontal curve $\gamma$ is called radial curve if it can be continuously and monotonically reparametrized as

$$
s \mapsto \phi(s, p), \quad \text { for } p \in \mathfrak{H} \backslash \text { (t-axis). }
$$

Radial curves can be explicitly computed,

$$
\phi(s, z, t)=\left(s z e^{-\frac{\mathrm{i} t}{|z|^{2}} \log s}, s^{2} t\right), \quad \text { for } z \in \mathbb{C} \backslash\{0\}, t \in \mathbb{R} ;
$$

see [6. Example 4.3].

If $(z, t) \in \partial B(0,1) \backslash\{z=0\}$, we have further that

$$
\gamma(\zeta)=\phi(s(\zeta),(z, t)) \Leftrightarrow \frac{\gamma_{I}(\zeta)}{\|\gamma(\zeta)\|_{H}} e^{\mathrm{i} \frac{\gamma_{3}(\zeta)}{\left|\gamma_{I}(\zeta)\right|^{2}} \log \|\gamma(\zeta)\|_{H}}=z, \frac{\gamma_{3}(\zeta)}{\|\gamma(\zeta)\|_{H}^{2}}=t
$$

Remark 2.2. The subfamily of radial curves for which $t /|z|^{2}=c$ coincides with the Legendrian foliation of the Heisenberg cone $C_{c}=\left\{(z, t): t=c|z|^{2}\right\}$. The terminology, which has been used before, for instance, in [9, 4.3.2], is motivated by the fact that Heisenberg cones are invariant under the intrinsic dilations $\delta_{r}(z, t)=$ $\left(r z, r^{2} t\right), r>0$.

It is known that there exists a positive Radon measure $\sigma$ on the Korányi unit sphere $\partial B(0,1)$ such that

$$
\int_{\mathfrak{H}} h(p) \mathrm{d} \mathcal{L}^{3}=\int_{\partial B(0,1)} \int_{0}^{\infty} h(\phi(s, q)) s^{3} \mathrm{~d} s \mathrm{~d} \sigma(q), \quad \text { for all } h \in L^{1}(\mathfrak{H}) .
$$

This formula shows that for $A \subseteq \mathfrak{H}$, we have

$\mathcal{L}^{3}(A)=0 \quad$ if and only if $\quad \mathcal{L}^{1}(\{s \in \mathbb{R}: \phi(s, q) \in A\})=0$, for $\sigma$ a.e. $q \in \partial B(0,1)$.

Radial curves were first studied by Korányi and Reimann [14, and then by Balogh and Tyson [6]. See for instance [9, 5.4] for a discussion and proofs of the mentioned properties.

The 4-modulus of the family of all radial curves that connect the boundary spheres of a Kóranyi annulus in $\mathfrak{H}$ was first computed in [14. In Proposition 2.3. we state a generalisation of this result that allows to compute the modulus also for subfamilies of radial curves. The modulus on the Heisenberg group is defined in the standard way, and it is a special instance of Fuglede's modulus, which is given 
on an arbitrary metric measure space. The additional structure of $\mathfrak{H}$ can be used to derive the formula

$$
\int_{\gamma} \rho \mathrm{d} s=\int_{a}^{b} \rho(\gamma(s))\left|\dot{\gamma}_{I}(s)\right| \mathrm{d} s
$$

for the line integral of a Borel function along a horizontal curve $\gamma=\left(\gamma_{1}+\mathrm{i} \gamma_{2}, \gamma_{3}\right)=$ $\left(\gamma_{I}, \gamma_{3}\right):[a, b] \rightarrow \mathfrak{H}$.

Proposition 2.3. Let $0<a<b$ and let $A^{\prime}$ be a subset of $\partial B(0,1) \backslash\{z=0\}$. Denoting

$$
A=\left\{\phi(s, q): s \in(a, b), q \in A^{\prime}\right\}
$$

we have

$$
\operatorname{Mod}\left(\left\{\left.\phi(\cdot, q)\right|_{(a, b)}: q \in A^{\prime}\right\}\right)=\int_{A} \rho_{0}^{4} \mathrm{~d} \mathcal{L}^{3}=\left(\log \frac{b}{a}\right)^{-3} \int_{A^{\prime}}|z|^{4} \mathrm{~d} \sigma(z, t),
$$

for

$$
\rho_{0}=\frac{1}{\log \left(\frac{b}{a}\right)}\left|\nabla_{0} \log u\right|_{0} \chi_{A},
$$

provided that $A$ and $A^{\prime}$ are measurable. Then we have, in particular,

$$
\operatorname{Mod}\left(\left\{\left.\phi(\cdot, q)\right|_{(a, b)}: q \in A^{\prime}\right\}\right)=0 \quad \Leftrightarrow \quad \sigma\left(A^{\prime}\right)=0 \quad \Leftrightarrow \quad \mathcal{L}^{3}(A)=0 .
$$

In particular, if $\Gamma_{0}$ is the full family of all radial curves in $S_{a, b}$ that connect the two boundary components, we have

$$
\operatorname{Mod}\left(\Gamma_{0}\right)=\int_{S_{a, b}} \rho_{0}^{4} \mathrm{~d} \mathcal{L}^{3}=\pi^{2}\left(\log \left(\frac{b}{a}\right)\right)^{-3} .
$$

Remark 2.4. For the expression of $\rho_{0}$ in logarithmic coordinates, see (1.2).

The proof of Proposition 2.3 is left as an exercise to the interested reader with the following hint. The modulus can be computed analogously as in [14] or [4, where the special case of the full family of all radial curves was considered. The other identities are consequences of the integration formula (2.2). The last statement follows by considering $A^{\prime}=\partial B(0,1) \backslash\{z=0\}$.

Let now $\Gamma$ be the family of all curves in $S_{a, b}$ that connect the boundary components. The density

$$
\rho_{0}=\frac{1}{\log \left(\frac{b}{a}\right)}\left|\nabla_{0} \log u\right|_{0} \chi_{S_{a, b}}
$$

which is extremal for the subfamily $\Gamma_{0}$ of radial curves, is admissible and extremal also for this larger family, that is,

$$
\operatorname{Mod}(\Gamma)=\int_{S_{a, b}} \rho_{0}^{4} \mathrm{~d} \mathcal{L}^{3} .
$$

For a curve $\gamma \in \Gamma$, we have in general $\int_{\gamma} \rho_{0} \mathrm{~d} s \geq 1$. The next lemma shows that equality in this estimate characterizes the radial curve.

Lemma 2.5. Let $\gamma \in \Gamma$ be a horizontal curve inside $S_{a, b}$ joining the two boundary components. Then $\gamma$ is a radial curve if and only if $\int_{\gamma} \rho_{0} \mathrm{~d} s=1$. 
Proof. Let $\gamma:[a, b] \rightarrow \bar{S}_{a, b}$ be a horizontal curve with $\|\gamma(a)\|_{H}=a,\|\gamma(b)\|_{H}=b$, and $\gamma(s) \in S_{a, b}$ for all $s \in(a, b)$. We denote by $\gamma_{I}$ the projection of $\gamma$ to $\mathbb{C} \times\{0\}$. Then we find

$$
\begin{aligned}
\int_{\gamma} \rho_{0} \mathrm{~d} s & =\frac{1}{\log \left(\frac{b}{a}\right)} \int_{a}^{b}\left|\left(\nabla_{0} \log u\right)(\gamma(s))\right|_{0}\left|\dot{\gamma}_{I}(s)\right| \mathrm{d} s \\
& =\frac{1}{\log \left(\frac{b}{a}\right)} \int_{a}^{b} \frac{1}{u(\gamma(s))}\left|\left(\nabla_{0} u\right)(\gamma(s))\right|_{0}|\dot{\gamma}(s)|_{0} \mathrm{~d} s \\
& \geq \frac{1}{\log \left(\frac{b}{a}\right)} \int_{a}^{b}\left|\frac{(u \circ \gamma)^{\prime}(s)}{u \circ \gamma(s)}\right| \mathrm{d} s \\
& \geq \frac{1}{\log \left(\frac{b}{a}\right)}|\log (u(\gamma(b)))-\log (u(\gamma(a)))|=1 .
\end{aligned}
$$

Note that the horizontality of $\gamma=\left(\gamma_{1}, \gamma_{2}, \gamma_{3}\right)$ implies

$$
(u \circ \gamma)^{\prime}(s)=X u(\gamma(s)) \dot{\gamma}_{1}(s)+Y u(\gamma(s)) \dot{\gamma}_{2}(s)=\left\langle\nabla_{0} u(\gamma(s)), \dot{\gamma}(s)\right\rangle_{0},
$$

at points of differentiability, thus equality holds in the above inequality if and only if

$$
\left|\nabla_{0} u(\gamma(s))\right|_{0}|\dot{\gamma}(s)|_{0}=\left\langle\nabla_{0} u(\gamma(s)), \dot{\gamma}(s)\right\rangle_{0}
$$

for almost all $s \in[a, b]$ and $(u \circ \gamma)^{\prime}(s)>0$ for almost all $s \in[a, b]$. Condition (2.6) is equivalent to $\dot{\gamma}(s)$ being parallel to $\nabla_{0} u(\gamma(s))$ and reparametrization (for instance according to arc length) shows that curves with this property are exactly the radials.

The upper and lower vertical axis can be interpreted as a degenerate radial curve (corresponding to the north pole $q=(0,0,1)$ and south pole $q=(0,0,-1)$ in the Korányi sphere, respectively). Even though it is not a horizontal curve, it is convenient to include it in the following discussion.

Definition 2.6. We denote by

$$
\Gamma_{0}^{*}=\Gamma_{0} \cup\left\{s \mapsto \phi(s,(0,0,1)):=\left(0,0, s^{2}\right)\right\} \cup\left\{s \mapsto \phi(s,(0,0,-1))=\left(0,0,-s^{2}\right)\right\}
$$

the family of generalised radials.

As we consider maps $f: S_{a, b} \rightarrow S_{a^{k}, b^{k}}$ in Theorem 1.5, we study not only the curve families $\Gamma_{0}, \Gamma, \Gamma_{0}^{*}$ in the source space, but also the corresponding families $\Gamma_{0}^{\prime}, \Gamma^{\prime}, \Gamma_{0}^{*^{\prime}}$ in the target domain. It turns out that the considered minimisers push the extremal density $\rho_{0}$ forward to an extremal density in the target, as discussed in the next section.

2.1.2. Minimisers preserve the family of generalised radials. Modulus inequalities for quasiconformal maps on the Heisenberg group can be proved using the analytic properties of such maps. A quasiconformal map $f: \Omega \rightarrow \Omega^{\prime}$ between domains in $\mathfrak{H}$ is almost everywhere differentiable in the sense of Pansu [16]. Pansu calls a map $f$ differentiable at $p$ if

$$
\delta_{\frac{1}{h}} \circ L_{f(p)^{-1}} \circ f \circ \delta_{h}
$$

converges uniformly on $\bar{B}(0,1)$ to grading preserving group homomorphism as $h \rightarrow$ 0 (here $L_{q}$ denotes left translation with respect to the group law $*$ ). We call the limit map the Pansu derivative of $f$ at $p$; it can also be understood via the exponential 
map as a homomorphism of the underlying Lie algebra $\mathfrak{h}$. The Pansu derivative of a quasiconformal map is almost everywhere given by

$$
D f=\left(\begin{array}{cc}
D_{H} f & 0 \\
0 & \operatorname{det} D_{H} f
\end{array}\right), \quad \text { where } D_{H} f=\left(\begin{array}{cc}
X f_{1} & Y f_{1} \\
X f_{2} & Y f_{2}
\end{array}\right)
$$

with respect to the basis $\left\{X, Y, T=\partial_{t}\right\}$; see for instance [10]. Following the standard notation in Euclidean spaces [18, we write

$\left\|D_{H} f(p)\right\|=\sup _{v \in H_{p} \mathfrak{H}:|v|_{0}=1}\left|D_{H} f(p) v\right|_{0} \quad$ and $\quad \ell\left[D_{H} f(p)\right]=\inf _{v \in H_{p} \mathfrak{H}:|v|_{0}=1}\left|D_{H} f(p) v\right|_{0}$.

In complex notation, we obtain

$$
\left\|D_{H} f\right\|=\left|Z f_{I}\right|+\left|\bar{Z} f_{I}\right| \text { and } \ell\left[D_{H} f\right]=|| Z f_{I}|-| \bar{Z} f_{I}|| .
$$

Here we restrict our discussion to maps that are orientation preserving in the sense that

$$
\operatorname{det} D_{H} f=\left|Z f_{I}\right|-\left|\bar{Z} f_{I}\right|>0, \quad \text { almost everywhere. }
$$

Note that the orientation of a map can be changed by composing with a conformal reflection.

Definition 2.7. Consider an orientation preserving quasiconformal map $f: \Omega \rightarrow$ $\Omega^{\prime}$ between domains in $\mathfrak{H}$ and a Borel function $\rho: \Omega \rightarrow[0,+\infty]$. Let $B$ be a Borel set with $\mathcal{L}^{3}(B)=0$ and such that $f$ is Pansu differentiable in $\Omega \backslash B$, the horizontal derivatives $Z f_{I}$ and $\bar{Z} f_{I}$ exist and satisfy $\left|Z f_{I}\right|-\left|\bar{Z} f_{I}\right|>0$ everywhere in $\Omega \backslash B$. We define the push-forward of $\rho: \Omega \rightarrow[0,+\infty]$ under $f$ as

$$
\rho^{\prime}: \Omega^{\prime} \rightarrow[0,+\infty], \quad \rho^{\prime}(\zeta):= \begin{cases}\frac{\rho}{\left|Z f_{I}\right|-\left|\bar{Z} f_{I}\right|} \circ f^{-1}(\zeta), & \text { if } \zeta \in \Omega^{\prime} \backslash f(B), \\ \infty, & \text { if } \zeta \in f(B), \\ 0, & \text { if } \zeta \in \mathfrak{H} \backslash \Omega^{\prime}\end{cases}
$$

Note that the definition of $\rho^{\prime}$ depends on the choice of $B$, but all the choices lead to densities that agree almost everywhere and have the correct admissibility properties.

The push-forward density is tailored so that if $\rho$ is admissible for a curve family $\Gamma$ in $\Omega$, the density $\rho^{\prime}$ will be admissible for $f(\Gamma)$ since $\int_{f \circ \gamma} \rho^{\prime} \mathrm{d} s \geq \int_{\gamma} \rho \mathrm{d} s$ for all locally rectifiable curves in $\Gamma$. This idea goes back to Grötzsch and Ahlfors. The notion of push-forward density provides information on the image of radial curves under a minimiser of the distortion functional in Theorem 1.5

Proposition 2.8. Let $f \in \mathcal{F}$ be a map that satisfies

$$
\operatorname{Mod}\left(\Gamma_{0}^{\prime}\right)=\int_{S_{a, b}} K_{f}^{2} \rho_{0}^{4} \mathrm{~d} \mathcal{L}^{3} .
$$

Then $f\left(\Gamma_{0}^{*}\right)=\Gamma_{0}^{* *}$.

Proof. The integral in the statement can be rewritten as

$$
\int_{S_{a, b}} K_{f}^{2} \rho_{0}^{4} \mathrm{~d} \mathcal{L}^{3}=\int_{S_{a^{k}, b^{k}}} \rho_{0}^{\prime 4} \mathrm{~d} \mathcal{L}^{3},
$$

where $\rho_{0}^{\prime}$ denotes the push-forward density of $\rho_{0}$ under $f$. Since $\rho_{0}^{\prime}$ is admissible for $\Gamma_{0}^{\prime}$, it follows that it is in fact extremal. Another extremal density for the 
same family is provided by $\tilde{\rho}_{0}=k^{-1}\left(\log \frac{b}{a}\right)^{-1}\left|\nabla_{0} \log u\right|_{0} \chi_{S_{a^{k}, b^{k}}}$. We conclude by Proposition 3.4 in the Appendix that

$$
\tilde{\rho}_{0}=\rho_{0}^{\prime}, \quad \text { almost everywhere in } S_{a^{k}, b^{k}} .
$$

By (2.3), it follows for $\sigma$ almost all $q \in \partial B(0,1)$ that $\tilde{\rho}_{0}(\phi(s, q))=\rho_{0}^{\prime}(\phi(s, q))$ for almost every $s$. Moreover, since quasiconformal maps are absolutely continuous on 4-almost every curve [5], we have that $f^{-1}(\phi(\cdot, q))$ is horizontal for $\sigma$ almost all $q$. In particular,

$$
\begin{aligned}
1=\int_{\phi(\cdot, q) \cap S_{a^{k}, b^{k}}} \tilde{\rho}_{0} \mathrm{~d} s & =\int_{\phi(\cdot, q) \cap S_{a^{k}, b^{k}}} \rho_{0}^{\prime} \mathrm{d} s \\
& \geq \int_{f^{-1}\left(\phi(\cdot, q) \cap S_{a^{k}, b^{k}}\right)} \rho_{0} \mathrm{~d} s \geq 1, \quad \text { for } \sigma \text { a.e. } q .
\end{aligned}
$$

We conclude by Lemma 2.5 that for $\sigma$ almost all $q \in \partial B(0,1)$, the curve $f^{-1}(\phi(\cdot, q) \cap$ $\left.S_{a^{k}, b^{k}}\right)$ is a radial curve. We reparametrise it by $\left\|f^{-1}(\phi(s, q))\right\|_{H}$ to bring it in standard form. In conclusion, there exists a $\sigma$ full measure set $A \subseteq \partial B(0,1)$ such that for all $q \in A$, we have

$$
f^{-1}(\phi(s, q))=\phi(\underline{s}(s, q), \underline{q}(s, q)), \quad \text { for all } s \in\left[a^{k}, b^{k}\right],
$$

with

$$
\underline{s}(s, q)=\left\|f^{-1}(\phi(s, q))\right\|_{H}
$$

and

$$
\underline{q}(s, q)=\phi\left(\frac{1}{a}, f^{-1}\left(\phi\left(a^{k}, q\right)\right)\right) .
$$

Here the function $\phi$ is defined by

$$
\phi(s,(z, t))= \begin{cases}\left(s z e^{-i \frac{t}{|z|^{2}} \log s}, s^{2} t\right), & z \neq 0, \\ \left(0, s^{2} t\right) & z=0,\end{cases}
$$

as in the definition for the generalised radials. Note that $\phi$ is continuous on $(0,+\infty) \times(\mathfrak{H} \backslash\{0\})$.

Now we wish to show for an arbitrary point $q$ in $\partial B(0,1) \backslash A$ that $f^{-1} \circ \phi(\cdot, q)$ has to be a generalised radial curve. Indeed, since $A$ is a full measure set, there must exist a sequence $\left(q_{n}\right)_{n} \subseteq A$ with $q_{n} \rightarrow q$ as $n \rightarrow \infty$. Yet then, continuity of the Korányi norm, as well as of the functions $\phi$ and $f^{-1}$, implies that

$$
f^{-1}(\phi(s, q))=\phi(\underline{s}(s, q), \underline{q}(s, q)), \quad \text { for all } s \in\left[a^{k}, b^{k}\right],
$$

In particular, we see that $f^{-1} \circ \phi(\cdot, q)$ is a curve in the family $\Gamma_{0}^{*}$. Thus we have proved that $f^{-1}\left(\Gamma_{0}^{\prime *}\right) \subseteq \Gamma_{0}^{*}$. Since $f^{-1}\left(S_{a^{k}, b^{k}}\right)=S_{a, b}$, and annuli are foliated by the generalised radial curves, we conclude that $f^{-1}\left(\Gamma_{0}^{*}\right)=\Gamma_{0}^{*}$.

Proposition 2.8 shows that the minimisers in Theorem 1.5 are of the form

$$
\begin{aligned}
& f\left(s z e^{-\frac{t}{|z|^{2}} \log s}, s^{2} t\right)=\left(\zeta_{q}(s) z^{\prime} e^{-\frac{t^{\prime}}{\left|z^{\prime}\right|^{2}} \log \zeta_{q}(s)}, \zeta_{q}(s)^{2} t^{\prime}\right) \\
& \text { for some } q^{\prime}=q^{\prime}(q)=\left(z^{\prime}, t^{\prime}\right) \in \partial B(0,1)
\end{aligned}
$$

with $\zeta_{q}:[a, b] \rightarrow\left[a^{k}, b^{k}\right]$ for $\sigma$ almost all $q=(z, t) \in \partial B(0,1)$. 
2.1.3. Equivalent reformulations of the problem. We are looking for maps that realise equality in (1.3) in Theorem 1.5. The goal of this section is to give an equivalent formulation of the problem, which will be a key to its solution.

As before, let $\rho_{0}$ be as in (2.5), $\Gamma_{0}$ and $\Gamma_{0}^{\prime}$ the family of radial curves in $S_{a, b}$ and $S_{a^{k}, b^{k}}$, respectively, and $\Gamma, \Gamma^{\prime}$ the larger families consisting of all curves that connect the boundary components of $S_{a, b}$ and $S_{a^{k}, b^{k}}$, respectively.

Proposition 2.9. For a map $f \in \mathcal{F}$, the following are equivalent:

(1) $f$ satisfies

$$
k^{-3}=\mathfrak{M}\left(f_{k}, \rho_{0}\right)=\mathfrak{M}\left(f, \rho_{0}\right),
$$

(2) $f$ satisfies

$$
\operatorname{Mod}\left(\Gamma^{\prime}\right)=\operatorname{Mod}\left(\Gamma_{0}^{\prime}\right)=\int_{S_{a, b}} K_{f}^{2} \rho_{0}^{4} d \mathcal{L}^{3}
$$

(3) $f$ satisfies

$$
\operatorname{Mod}\left(f\left(\Gamma_{0}\right)\right)=\int_{S_{a, b}} K_{f}^{2} \rho_{0}^{4} d \mathcal{L}^{3}
$$

Proof. (11) is equivalent to (2) since the radial stretch map $f_{k}$ has the property that

$$
\int_{S_{a, b}} K_{f_{k}}^{2} \rho_{0}^{4} \mathrm{~d} \mathcal{L}^{3}=\operatorname{Mod}\left(\Gamma_{0}^{\prime}\right)
$$

and the radial curves satisfy

$$
\operatorname{Mod}\left(\Gamma_{0}^{\prime}\right)=\operatorname{Mod}\left(\Gamma^{\prime}\right)
$$

The implication from (2) to (3) is based on the fact that a map $f$ that satisfies (2) maps the family of generalised radials $\Gamma_{0}^{*}$ onto the generalised radials $\Gamma_{0}^{*^{\prime}}$ by Proposition 2.8. The $t$-axis and its possible images and preimages under $f$ form a family of vanishing modulus, so that they can be safely ignored.

Finally, assume that (3) holds. For all maps $f$ in the class $\mathcal{F}$, we always have by the considerations in 4 that

$$
\operatorname{Mod}\left(f\left(\Gamma_{0}\right)\right) \leq \operatorname{Mod}\left(\Gamma^{\prime}\right) \text { and } \operatorname{Mod}\left(\Gamma^{\prime}\right)=\operatorname{Mod}(f(\Gamma)) \leq \int_{S_{a, b}} K_{f}^{2} \rho_{0}^{4} \mathrm{~d} \mathcal{L}^{3},
$$

so the validity of (3) implies that the above inequalities must in fact be equalities and hence $\operatorname{Mod}\left(\Gamma^{\prime}\right)=\int_{\Omega} K_{f}^{2} \rho_{0}^{4} \mathrm{~d} \mathcal{L}^{3}$ and (2) holds.

2.1.4. Horizontal derivatives. As mentioned in the introduction, the components of a quasiconformal map on the Heisenberg group belong to a horizontal Sobolev space. This means that they have distributional derivatives in direction of all horizontal left-invariant vector fields (in particular $X, Y, Z, \bar{Z}$ ). Moreover, the usual Lie derivatives and partial derivatives in these directions exist almost everywhere and agree with the distributional derivatives [13, 3.1].

Definition 2.10. Let $V$ be a smooth vector field on a domain $\Omega \subseteq \mathfrak{H}$ and consider its flow, which exists in a neighborhood of every point in $\Omega$ with unique integral curves $s \mapsto \gamma_{q}(s)$, satisfying

$$
\left\{\begin{array}{l}
\dot{\gamma}_{q}(s)=V_{\gamma_{q}(s)} \\
\gamma_{q}(0)=q
\end{array}\right.
$$


The Lie derivative of a function $h: \Omega \rightarrow \mathbb{R}$ along $V$ in a point $p \in \Omega$ is defined as

$$
(V h)(p)=\lim _{s \rightarrow 0} \frac{h\left(\gamma_{p}(s)\right)-h(p)}{s}
$$

provided that this limit exists.

In connection with the minimisation problem, we deal with radial curves. They are the flow of a horizontal, but not left invariant vector field. The radial curves $\gamma_{p}(s)=\phi(s, p), p \in \partial B(0,1) \backslash\{z=0\}$, are integral curves of the vector field

$$
U=\frac{\nabla_{0} u}{\left|\nabla_{0} u\right|^{2}}=\frac{u^{2}}{|z|^{2}} \nabla_{0} u
$$

where

$$
\nabla_{0} u=u^{-3}\left(\left(|z|^{2}-\mathrm{i} t\right) z Z+\left(|z|^{2}+\mathrm{i} t\right) \bar{z} \bar{Z}\right), \quad u=\|\cdot\|_{H}
$$

see [14, (3.11), (4.3)].

Assume now that $h: \Omega \rightarrow \mathbb{R}$ is defined on an open set $\Omega$ in $\mathfrak{H}$ and $p=\phi\left(s_{0}, q\right) \in$ $\Omega$. It follows directly from the definitions that the Lie derivative $U h$ with $U$ as in (2.10) exists at $p$ if and only if $s \mapsto h \circ \phi\left(s_{0}+s, q\right)$ is differentiable at $s=0$, and in this case

$$
(U h)(p)=(U h)\left(\phi\left(s_{0}, q\right)\right)=(h \circ \phi(\cdot, q))^{\prime}\left(s_{0}\right) .
$$

The components of quasiconformal maps are examples of functions that admit a Lie derivative along $U$ almost everywhere.

Lemma 2.11. Let $\Omega, \Omega^{\prime} \subseteq \mathfrak{H}$ be domains and $f=\left(f_{1}, f_{2}, f_{3}\right): \Omega \rightarrow \Omega^{\prime}$ a quasiconformal map. Then for $\mathcal{L}^{3}$ almost all $p \in \Omega$, the Lie derivatives $U f_{i}, i \in\{1,2,3\}$, exist at $p$.

Proof. The property is local, so we may assume that our domain is of the form

$$
\Omega=\left\{\phi(s, q): s \in(a, b), q \in A^{\prime}\right\}
$$

for an open set $A^{\prime} \in \partial B(0,1) \backslash\{0\}$ and numbers $0<a<b$. Since $f$ is quasiconformal, it is absolutely continuous with respect to $d_{H}$ on 4-almost every curve in the family $\left\{\left.\phi(\cdot, q)\right|_{(a, b)}: q \in A^{\prime}\right\}$. It follows from Proposition 2.3 that the set $E$ inside $\Omega$ which is foliated by the exceptional curves (along which $f$ is not absolutely continuous) is of vanishing $\mathcal{L}^{3}$ measure. Since absolutely continuous curves are horizontal and in particular differentiable almost everywhere, we find by another application of the integral formula (2.2) that

$$
\mathcal{L}^{3}\left(\left\{\phi\left(s_{0}, q\right):(f \circ \phi(\cdot, q))^{\prime}\left(s_{0}\right) \text { does not exist }\right\}\right)=0 .
$$

For points $p$ (and corresponding $q, s_{0}$ ) outside this exceptional set, the tangent vector $(f \circ \phi(\cdot, q))^{\prime}\left(s_{0}\right)$ exists and hence also the Lie derivatives of all components of $f$.

2.1.5. The minimal stretching property. In this section, we adopt a broader viewpoint and derive a necessary condition for a rather general class of distortion minimisers. In this sense we state a converse of [4, Proposition 5].

Proposition 2.12. Assume that $f: \Omega \rightarrow \Omega^{\prime}$ is an orientation preserving quasiconformal map between domains in $\mathfrak{H}$ and $\Gamma_{0}$ a family of locally rectifiable curves in $\Omega$. Suppose that there exists $\rho_{0} \in \operatorname{adm}\left(\Gamma_{0}\right)$ such that

$$
\operatorname{Mod}\left(f\left(\Gamma_{0}\right)\right)=\int_{\Omega} K_{f}^{2}(p) \rho_{0}^{4}(p) d \mathcal{L}^{3}(p) .
$$


Assume further that

(a) $\int_{f \circ \gamma} \rho_{0}^{\prime} \mathrm{d} s=1$ for 4 -almost all $\gamma \in \Gamma_{0}$,

(b) $\rho_{0}(p) \neq 0$ for all $p \in \Omega$,

(c) for all sets $A \subseteq \Omega$ with $\mathcal{L}^{3}(A)=0$, we have

$$
\operatorname{Mod}\left(\left\{\gamma \in \Gamma_{0}: \mathcal{L}^{1}(\{s: \gamma(s) \in A\})>0\right\}\right)=0
$$

(Fubini-type property).

Then we have for 4-almost all $\gamma \in \Gamma_{0}$ :

$$
\begin{aligned}
\left(\left|Z f_{I}(\gamma(s))\right|-\left|\bar{Z} f_{I}(\gamma(s))\right|\right)\left|\dot{\gamma}_{I}(s)\right|= & \left|(f \circ \gamma)_{I}^{\prime}(s)\right| \\
& \text { for almost all } s \text { in the domain of } \gamma .
\end{aligned}
$$

We say that a curve family $\Gamma_{0}$ with the property (2.11) satisfies the minimal stretching property (MSP) for the map $f$. The terminology is motivated by the observation that the inequality

$$
\ell\left[D_{H} f(\gamma(s))\right]|\dot{\gamma}(s)|_{0}=\left(\left|Z f_{I}(\gamma(s))\right|-\left|\bar{Z} f_{I}(\gamma(s))\right|\right)\left|\dot{\gamma}_{I}(s)\right| \leq\left|(f \circ \gamma)_{I}^{\prime}(s)\right|,
$$

for almost all $s$ in the domain of $\gamma$, holds for arbitrary horizontal curves along which $f$ is almost everywhere differentiable. The curves with the MSP are precisely those that point in the direction along which $f$ stretches the least possible amount. We emphasise that the MSP is invariant under regular reparametrisations of the curve $\gamma$.

Proof of Proposition 2.12, Let $E$ be the exceptional set in $\Omega$ where $f$ is not differentiable in the sense of Pansu or has a singular derivative. This is a set of zero measure and by condition (c), we may assume that all curves $\gamma$ in $\Gamma_{0}$ satisfy $\gamma(s) \in \Omega \backslash E$ for almost every $s$ in their domain. Since $f$ is quasiconformal, it is absolutely continuous on 4 -almost curves in $\Gamma_{0}$ and we have $\int_{f \circ \gamma} \rho_{0}^{\prime} \mathrm{d} s=1$ by assumption (a). Let $\gamma$ be an arbitrary curve of this type. It is differentiable almost everywhere both in the usual sense and in the sense of Pansu with derivative

$$
\dot{\gamma}(s)=\dot{\gamma}_{1}(s) X_{\gamma(s)}+\dot{\gamma}_{2}(s) Y_{\gamma(s)} ;
$$

see [16, Proposition 4.1]. We may assume, without loss of generality, that $\gamma$ is parameterised according to arc length and that $f \circ \gamma$ is absolutely continuous. By the chain rule for Pansu derivatives, in almost every point $s$, we find

$$
(f \circ \gamma)^{\prime}(s)=D_{H} f(\gamma(s)) \dot{\gamma}(s) .
$$

Here the horizontal structure is reflected both in the fact that $\gamma$ and $f \circ \gamma$ are tangential to the horizontal distribution, as well as in the fact that the Pansu derivative of $f$ preserves the grading. The identity (2.12) yields

$$
\left|(f \circ \gamma)^{\prime}(s)\right|_{0} \geq \ell\left[D_{H} f(\gamma(s))\right] \cdot|\dot{\gamma}(s)|_{0},
$$

or, equivalently in complex notation,

$$
\left|(f \circ \gamma)_{I}^{\prime}(s)\right| \geq\left(\left|Z f_{I}(\gamma(s))\right|-\left|\bar{Z} f_{I}(\gamma(s))\right|\right) \cdot\left|\dot{\gamma}_{I}(s)\right|
$$


for almost all $s \in I=[0, \operatorname{length}(\gamma)]$. To prove the claim, we consider the pushforward density $\rho_{0}^{\prime} \in \operatorname{adm}\left(f\left(\Gamma_{0}\right)\right)$ as in (2.8). Now, by (2.13),

$$
\begin{aligned}
1=\int_{f \circ \gamma} \rho_{0}^{\prime} \mathrm{d} s & =\int_{I}\left(\frac{\rho_{0}}{\left|Z f_{I}\right|-\left|\bar{Z} f_{I}\right|} \circ f^{-1}\right)(f(\gamma(s))) \cdot\left|(f \circ \gamma)_{I}^{\prime}(s)\right| d s \\
& =\int_{I} \frac{\rho_{0}(\gamma(s))}{\left|Z f_{I}(\gamma(s))\right|-\left|\bar{Z} f_{I}(\gamma(s))\right|} \cdot\left|(f \circ \gamma)^{\prime}{ }_{I}(s)\right| d s \\
& \geq \int_{I} \rho_{0}(\gamma(s))\left|\dot{\gamma}_{I}(s)\right| d s \\
& =\int_{\gamma} \rho_{0} \mathrm{~d} s \geq 1 .
\end{aligned}
$$

This gives

$$
\left|(f \circ \gamma)^{\prime}(s)\right|=\left(\left|Z f_{I}(\gamma(s))\right|-\left|\bar{Z} f_{I}(\gamma(s))\right|\right) \cdot\left|\dot{\gamma}_{I}(s)\right| \text { for almost all } s .
$$

2.2. Conservation of spheres. Proposition 2.12 applies to the minimisers in Theorem 1.5 and shows that the radial curves have the minimal stretching property for such maps. We know further that the family of generalised radials in $S_{a, b}$ is mapped to the corresponding family in $S_{a^{k}, b^{k}}$ under a minimiser $f$. In this section, we combine these facts with the contact property in order to deduce that minimisers are sphere-preserving.

2.2.1. The contact property. Quasiconformal maps on $\mathfrak{H}$ are weakly contact. That is, their distributional or usual derivatives satisfy the contact equations

$$
\left\{\begin{array}{l}
\bar{f}_{I} Z f_{I}-f_{I} Z \bar{f}_{I}=-\mathrm{i} Z f_{3}, \\
f_{I} \bar{Z} \bar{f}_{I}-\bar{f}_{I} \bar{Z} f_{I}=\mathrm{i} \bar{Z} f_{3}
\end{array}\right.
$$

from [12, p.335] almost everywhere in the domain. As in [12], it follows that

$$
Z\left(f_{3}+\mathrm{i}\left|f_{I}\right|^{2}\right)=2 \mathrm{i} \bar{f}_{I} Z f_{I} \quad \text { and } \quad \bar{Z}\left(f_{3}+\mathrm{i}\left|f_{I}\right|^{2}\right)=2 \mathrm{i} \bar{f}_{I} \bar{Z} f_{I}, \quad \text { almost everywhere. }
$$

We denote

$$
f_{I I}:=f_{3}+\mathrm{i}\left|f_{I}\right|^{2} .
$$

This expression will play a particular role in the following. For the moment, simply note that $\left|f_{I I}\right|=\|f\|_{H}^{2}$.

Equations (2.14) and (2.15) remain true if both sides are multiplied by some constant. Thus, they continue to hold, if $Z, \bar{Z}$ are replaced by $W, \bar{W}$, where

$$
W:=\frac{\left(|z|^{2}-\mathrm{i} t\right) z}{u(z, t)|z|^{2}} Z \quad \text { and } \quad \bar{W}:=\frac{\left(|z|^{2}+\mathrm{i} t\right) \bar{z}}{u(z, t)|z|^{2}} \bar{Z}
$$

for $z \neq 0$. As before, $u(z, t)=\sqrt[4]{|z|^{4}+t^{2}}$. The operators $W$ and $\bar{W}$ are chosen so that $U=W+\bar{W}$; see (2.10). A remark concerning the notation is in order here.

Remark 2.13. For the functions $h$ that we consider in the following, the horizontal derivatives $Z$ and $\bar{Z}$ in the distributional, Lie and directional sense exist and agree almost everywhere. Given complex-valued functions $a$ and $b$, we then write $(a Z+$ $b \bar{Z}) h$ to mean the function $a(p)(Z h)(p)+b(p)(\bar{Z} h)(p)$, which is defined up to a null set. Furthermore, for the considered functions $h$, also the Lie derivative of $h$ along the horizontal vector field $V:=a Z+b \bar{Z}$ exists almost everywhere. If, specifically, the function $h$ is defined through the components of a quasiconformal map $f$, then the fact that $f$ is Pansu-differentiable almost everywhere, yields that 
the Lie derivative of $h$ at $p$ in direction $V$ agrees with the usual directional derivative in direction $V_{p}$ and

$$
(V h)(p)=a(p)(Z h)(p)+b(p)(\bar{Z} h)(p) .
$$

See [13, 2.2] for a related discussion. This observation is a counterpart for the wellknown fact that the directional derivative at $p$ in direction $v$ of a map $h: \mathbb{R}^{n} \rightarrow \mathbb{R}$ which is totally differentiable at $p$ can be computed as $(h \circ \gamma)^{\prime}(0)$ for an arbitrary differentiable curve $\gamma$ with $\gamma(0)=p$ and $\dot{\gamma}(0)=v$.

2.2.2. Stretching in radial directions preserves spheres. The identity $U=W+\bar{W}$ relates $W, \bar{W}$ to the radial curves. If we consider a domain $\Omega$ foliated by radials and a map $f$ which has the minimal stretching property for the radials (as it is the case in our minimisation problem), then this relationship yields information on the $W$ and $\bar{W}$ derivatives of the components of $f$.

Proposition 2.14. Let $f: \Omega \rightarrow \Omega^{\prime}$ be a quasiconformal map on a domain

$$
\Omega=\left\{\phi(s, q): s \in(a, b), q \in A^{\prime}\right\}
$$

that is foliated by radial curves and assume that the map has the MSP for the radials inside the domain. Then

$$
\begin{array}{r}
\left|W f_{I}+\bar{W} f_{I}\right|=\left|W f_{I}\right|-\left|\bar{W} f_{I}\right| \quad \text { and }\left|W f_{I I}+\bar{W} f_{I I}\right|=\left|W f_{I I}\right|-\left|\bar{W} f_{I I}\right|, \\
\text { almost everywhere. }
\end{array}
$$

Proof. The crucial observation is that

$$
W_{\phi(s, q)}=\dot{\phi}_{I}(s, q) Z_{\phi(s, q)} \text { and } \quad \bar{W}_{\phi(s, q)}:=\dot{\bar{\phi}}_{I}(s, q) \bar{Z}_{\phi(s, q)} .
$$

The MSP then yields the first claim. The second one follows from (2.15).

If $f$ is a minimiser for the extremal problem in Theorem 1.5, one can derive quantitative information on the term that appears in Proposition 2.14 (depending on the amount of stretching).

Proposition 2.15. Let $f: S_{a, b} \rightarrow S_{a^{k}, b^{k}}$ be a quasiconformal map with the property that its push-forward density $\rho_{0}^{\prime}$ coincides almost everywhere with the extremal density for $\Gamma_{0}^{\prime}$ in the target. Then

$$
\left|W f_{I I}(p)\right|-\left|\bar{W} f_{I I}(p)\right|=2 k \frac{u(f(p))^{2}}{u(p)}, \quad \text { for almost every } p \in S_{a, b} .
$$

Proof. The equality $\rho_{0}^{\prime}=\widetilde{\rho}_{0}$ of the densities (as in Proposition 2.8) yields

$$
2\left|f_{I}(p)\right|\left(\left|Z f_{I}(p)\right|-\left|\bar{Z} f_{I}(p)\right|\right)=2 k \frac{|z| u(f(p))^{2}}{u(p)^{2}}, \quad \text { almost everywhere, }
$$

from where the claim follows.

Corollary 2.16. Let $f$ be a minimiser satisfying the assumptions of Theorem 1.5 , Then, for $\sigma$ almost all $q=(z, t) \in \partial B(0,1)$, we have

$$
f\left(s z e^{-\frac{t}{|z|^{2}} \log s}, s^{2} t\right)=\left(s^{k} z^{\prime} e^{-\frac{t^{\prime}}{\left|z^{\prime}\right|^{2}} \log s^{k}},\left(s^{k}\right)^{2} t^{\prime}\right)
$$

for some $q^{\prime}=q^{\prime}(q)=\left(z^{\prime}, t^{\prime}\right) \in \partial B(0,1)$. 
Proof. By quasiconformality of $f$ and the characterization of vanishing modulus for radial curves (Proposition 2.3), we know that $f \circ \phi(\cdot, q)$ is absolutely continuous for $\sigma$ almost all $q \in \partial B(0,1)$. Let us consider such $q$. As $f$ maps the family of generalised radials to generalised radials, we can further assume that $f(\phi(s, q))=\phi\left(\zeta_{q}(s), q^{\prime}(q)\right)$ for all $s \in[a, b]$ with a monotone function

$$
\zeta_{q}:[a, b] \rightarrow\left[a^{k}, b^{k}\right], \quad \zeta_{q}(a)=a^{k}, \quad \zeta_{q}(b)=b^{k} .
$$

Since $\zeta_{q}(s)=\|f \circ \phi(s, q)\|_{H}$, the function $\zeta_{q}$ is absolutely continuous. It has to satisfy a certain differential equation, which can be derived from Proposition 2.14 and Proposition 2.15. Indeed, a combination of these two propositions yields that for $\sigma$ almost all $q \in \partial B(0,1)$, we have

$$
\left|\left(f_{I I} \circ \phi(\cdot, q)\right)^{\prime}(s)\right|=\left|\left(U f_{I I}\right)(\phi(s, q))\right|=2 k \frac{u(f(p))^{2}}{u(p)}, \quad \text { for a.e. } s \in[a, b] .
$$

Inserting the formula for $f_{I I} \circ \phi(\cdot, q)$, this reads

$$
2 \zeta_{q}(s) \dot{\zeta}_{q}(s)=2 k \frac{\zeta_{q}(s)^{2}}{s}, \quad \text { for a.e. } s \in(a, b)
$$

By the absolute continuity and the boundary conditions of $\zeta_{q}$, we find by integration $\zeta_{q}(s)=s^{k}, s \in(a, b)$.

By continuity of $(s, q) \mapsto\|f \circ \phi(s, q)\|_{H}$, it follows that

$$
\|f \circ \phi(s, q)\|_{H}=\zeta_{q}(s)=s^{k} \quad \text { for all } q \in \partial B(0,1) .
$$

Corollary 2.17. Minimisers that satisfy the assumptions of Theorem 1.5 map spheres of radius $r \in[a, b]$ to spheres of radius $r^{k}$.

2.3. Spherical arcs are sent to spherical arcs. Radial curves have been introduced as flow curves of the vector fields $U=\frac{u^{2}}{|z|^{2}} \nabla_{0} u$; see (2.10). In this section, we consider the "orthogonal" vector field to $U$. By this we mean a horizontal vector field with the property that at each point $p$, the two tangent vectors $U_{p}$ and $V_{p}$ are orthogonal with respect to the scalar product $\langle\cdot, \cdot\rangle_{0}$ on $H_{p} \mathfrak{H}$ that makes $\left\{X_{p}, Y_{p}\right\}$ orthonormal. According to (2.10), the vector field $U$ is of the form

$$
a Z+\bar{a} \bar{Z}=\Re(a) X+\Im(a) Y,
$$

for some $a \in \mathbb{C}$, hence an orthogonal vector is given by

$$
i(a Z-\bar{a} \bar{Z})=-\Im(a) X+\Re(a) Y .
$$

More explicitly, we find

$$
V=\frac{\nabla_{0} u^{\perp}}{\left|\left(\nabla_{0} u\right)^{\perp}\right|^{2}}=\frac{u^{2}}{|z|^{2}}\left(\nabla_{0} u\right)^{\perp},
$$

where

$$
\left(\nabla_{0} u\right)^{\perp}:=\mathrm{i} u^{-3}\left(\left(|z|^{2}-\mathrm{i} t\right) z Z-\left(|z|^{2}+\mathrm{i} t\right) \bar{z} \bar{Z}\right) .
$$

Recall that $U$ is parallel to the horizontal normal on Korányi spheres $u \equiv r$. The tangents to the flow curves of $V$ are orthogonal to these normals with respect to $\langle\cdot, \cdot\rangle_{0}$, and, in fact, they form the Legendrian foliation of Korányi spheres. At each point $p$, the two vectors $U_{p}$ and $V_{p}$ form an orthonormal basis of $H_{p} \mathfrak{H}$, which is better adapted to the geometry of our minimisation problem than the standard basis $\left\{X_{p}, Y_{p}\right\}$. 
Definition 2.18. We call curves that are obtained by a reparametrisation of integral curves of $V$ spherical arcs.

We start by computing the 4-modulus of a family of spherical arcs and then continue with a discussion of how the minimisers behave along spherical arcs.

Proposition 2.19. Let $\gamma(s)=\left(\sqrt{\cos s} e^{i s / 2},-\sin (s)\right)$ be an arc on the Korányi unit sphere. Further, let $0<a<b$, and for each $r \in(a, b)$ let $A_{r}$ be a subset of $[0,2 \pi]$. Then the curve family

$$
\begin{aligned}
\Gamma_{A, a, b}=\left\{\delta_{r} \circ m_{\alpha} \circ \gamma: r \in(a, b),\right. & \left.\alpha \in A_{r}\right\}, \\
& \text { where } \delta_{r}(z, t)=\left(r z, r^{2} t\right), m_{\alpha}(z, t)=\left(e^{i \alpha} z, t\right),
\end{aligned}
$$

has modulus

$$
\operatorname{Mod}\left(\Gamma_{A, a, b}\right)=\frac{16 \int_{a}^{b} \frac{1}{r} \mathcal{L}^{1}\left(A_{r}\right) \mathrm{d} r}{\left(\int_{-\frac{\pi}{2}}^{\frac{\pi}{2}} \cos ^{-2 / 3}(s) \mathrm{d} s\right)^{3}} .
$$

Proof. The inequality $\geq$ follows by Hölder's inequality along standard lines. For the reverse inequality $\leq$, we use the density

$$
\rho_{0}\left(\delta_{r}\left(m_{\alpha}(\gamma(s))\right)=\frac{\left|\dot{\gamma}_{I}(s)\right|^{1 / 3}}{r \int_{-\frac{\pi}{2}}^{\frac{\pi}{2}}\left|\dot{\gamma}_{I}(s)\right|^{4 / 3} \mathrm{~d} s} .\right.
$$

Spherical arcs have a maximal stretching property for the minimisers of Theorem 1.5. in the sense that they are tangent to the directions of largest stretching of such maps.

Proposition 2.20. Let $f$ be a minimiser satisfying the assumptions of Theorem 1.5. Then $f$ has the maximal stretching property for the family of spherical arcs that belong to a Legendrian foliation of $\partial B(0, r), a<r<b$. Moreover, $f$ maps this family to the corresponding family in the target, apart from a possible exceptional family of modulus zero.

The maximal stretching property of spherical arcs will not be used for the proof of Theorem 1.5. but we decided to include it here for completeness and since it highlights the analogy between radials and arcs in the Heisenberg group on the one hand, and horizontal and vertical trajectories of quadratic differentials in Teichmüller theory on the other hand.

Proof of Proposition 2.20. Since $f$ is quasiconformal, it maps 4-almost every spherical arc in $S_{a, b}$ onto a horizontal curve. By the sphere-preserving property of $f$, the image curves have to be contained in spheres. Yet the only horizontal curves contained in a Korányi sphere are (concatenations and subcurves of) spherical arcs in its Legendrian foliation. Since two distinct arcs on a sphere intersect precisely in the north and south poles of the sphere, also the image curves have to intersect precisely at their start and end points. It remains to establish the maximal stretching property. At the same time, we will give an analytic proof for the fact that almost every spherical arc is mapped into a spherical arc. Recall that $f$ has the minimal stretching property for the family of radials. The radials are integral curves of the vector field $U$ and we have

$$
\left|D_{H} f(p) U_{p}\right|_{0}=\ell\left[D_{H} f(p)\right] \cdot\left|U_{p}\right|_{0}, \quad \text { for almost every } p .
$$


Here and in the following, we identify the various horizontal spaces $H_{p} \mathfrak{H}$ with $\mathbb{R}^{2}$ or with $\mathbb{C}$, using the left invariant frame. It is an exercise in linear algebra to verify for a linear map $A: \mathbb{R}^{2} \rightarrow \mathbb{R}^{2}$ that the unit vectors $v_{1}$ and $v_{2}$ (singular vectors) which realize the largest stretch $\|A\|$ and the smallest stretch $\ell[A]$, are orthogonal to each other; see for instance [18, 14]. Since $U_{p}$ and $V_{p}$ are orthogonal with respect to $\langle\cdot, \cdot\rangle_{0}$ in the horizontal plane, we conclude that the largest stretching happens in direction $V_{p}$. In almost every point $p$, the vector $U_{p}$ is not only a singular vector of $D_{H} f(p)$ (that is, an eigenvector of $D_{H} f(p)^{t} D_{H} f(p)$ ), but it is also an "eigenvector" of $D_{H} f(p)$ in the sense that $D_{H} f(p) U_{p}$ (as an element in $\left.H_{f(p)} \mathfrak{H}\right)$ is parallel to $U_{f(p)}$. This is a special property of the minimisers for our problem, which have to preserve the family of generalised radials. We conclude that almost everywhere,

$$
\begin{aligned}
\left\langle\left(\ell\left[D_{H} f(p)\right]\right)^{2} U_{p}, V_{p}\right\rangle_{0} & =\left\langle D_{H} f(p)^{t} D_{H} f(p) U_{p}, V_{p}\right\rangle_{0} \\
& =\left\langle D_{H} f(p) U_{p}, D_{H} f(p) V_{p}\right\rangle_{0} \\
& =\left\langle c(f, p) U_{f(p)}, D_{H} f(p) V_{p}\right\rangle_{0}
\end{aligned}
$$

for a non-vanishing scalar $c(f, p)$. We can assume that $D_{H} f(p)$ is non-singular and thus we find that $D_{h} f(p) V_{p}$ is orthogonal to $U_{f(p)}$ and parallel to $V_{f(p)}$ with respect to $\langle\cdot, \cdot\rangle_{0}$.

The curves belonging to the Legendrian foliations of spheres with radii $a<r<b$ foliate $S_{a, b}$, where the curves on one sphere intersect in their endpoints on the vertical axis. For 4-almost every curve $\gamma$ in this family, the image curve $f \circ \gamma$ is absolutely continuous with tangent parallel to $V_{f(\gamma(\cdot))}$ almost everywhere. Since $f \circ \gamma$ is absolutely continuous, it can be obtained by integration and we see that it agrees with an integral curve of $V$, that is, with a curve in a Legendrian foliation of a Korányi sphere. Here we use a similar Fubini type argument as for the radial curves; cf. Proposition 2.19.

Corollary 2.21. A minimiser $f$ has to preserve the vertical axis and send spherical arcs to spherical arcs. In particular, it cannot send a proper radial curve to the $t$ axis.

Proof. By the formula for the modulus in Proposition 2.19, we see that for almost every $r \in(a, b)$, arcs on $\partial B(0, r)$ corresponding to a full measure set of angles $\alpha \in(0,2 \pi)$ are sent to arcs. Similarly, as for the radial curves, we conclude by continuity of $f$ and the foliation given by spherical arcs, that in fact, all spherical arcs are sent to spherical arcs (to be precise, we use Lemma 3.5). Since all arcs on a given sphere meet the vertical axis, but a horizontal radial curve intersects only one arc on the sphere, it follows by the homeomorphism property of $f$ that the $t$-axis cannot be mapped to a horizontal radial curve, and instead, must be sent to the $t$-axis.

2.4. Rotation around the vertical axis. Curves in the Legendrian foliation of spheres have a slightly complicated expression in Cartesian coordinates or in Heisenberg polar coordinates. They take, however, a particularly simple form when expressed in logarithmic coordinates. In fact, this is a primary motivation to define coordinates this way. Recall that logarithmic coordinates are given by

$$
\Phi(\xi, \psi, \eta)=\left(\mathrm{i} \cos ^{1 / 2} \psi e^{\frac{\xi+\mathrm{i}(\psi-3 \eta)}{2}},-\sin \psi e^{\xi}\right) .
$$


Spherical arcs can then be parameterised simply as

$$
s \mapsto(\xi(s), \psi(s), \eta(s))=\left(\xi_{0}, s, \eta_{0}\right), \quad s \in\left[-\frac{\pi}{2}, \frac{\pi}{2}\right] .
$$

From the definition of the logarithmic coordinates, it is evident that the arcs corresponding to a fixed $\xi_{0}$ foliate the Korányi sphere of radius $e^{\xi_{0} / 2}$ with intersection points at the north and south poles. To prove that the so defined curves are horizontal, it is sufficient to observe that the contact form in logarithmic coordinates can be expressed as

$$
\tau=-e^{\xi}(\sin \psi d \xi+3 \cos \psi d \eta)
$$

see [17. Up to a reparametrisation, spherical arcs agree with the flow curves of $V$. As the parametrisation shows, they can be characterized in logarithmic coordinates by the conditions $\xi=$ const. and $\eta=$ const., which reminds us of the description of a planar circle in logarithmic coordinates.

Radial curves, on the other hand, can be parameterised as

$$
s \mapsto(\xi(s), \psi(s), \eta(s))=\left(s, \psi_{0}, \eta_{0}-\frac{\tan \psi_{0}}{3} s\right) .
$$

Their trace is of the form $\left\{(\xi, \psi, \eta): \psi=\psi_{0}, \eta+\frac{\tan \psi}{3} \xi=\eta_{0}\right\}$.

Remark 2.22. Since the minimisers $f$ from Theorem 1.5 have to preserve the vertical axis, such a map can be expressed in coordinates as $(\xi, \psi, \eta) \mapsto(\Xi, \Psi, H)$. A priori, the $\eta$-variable ranges only in an interval depending on $\psi$ of length $\frac{4 \pi}{3}$, but we will henceforth consider the covering space and allow the third variable to live in the entire $\mathbb{R}$. Such an extension is possible by $\frac{4 \pi}{3}$-periodicity of $\Xi, \Psi$ and $H$ in the $\eta$ variable, where in the case of $H$ the periodicity is to be understood in a generalised sense (modulo $\frac{4 \pi}{3}$ ).

Having established the behavior of minimisers in $\xi$ - and $\psi$-directions, we study in this section the behavior in the missing $\eta$-direction. The effect of $f$ on radial curves and spherical arcs leaves us with a functional equation for the $H$-component of $f$, which eventually provides the claimed form for $f$.

By (2.16), we already know for minimisers $f$ that $\Xi$ depends only on $\xi$ with

$$
\Xi(\xi, \psi, \eta)=k \xi \text {. }
$$

The fact that spherical arcs are sent to spherical arcs amounts to

$$
H(\xi, \psi, \eta)=H(\xi, \eta) .
$$

Indeed, since arcs can be parametrised as

$$
s \mapsto(\xi(s), \psi(s), \eta(s))=(\xi, s, \eta),
$$

we find that for fixed $(\xi, \eta)$ the function $H$ should not depend on $\psi$. Finally, since radials are sent to radials, for arbitrary fixed $(\psi, \eta)$, the function

$$
\xi \mapsto \Psi\left(\xi, \psi, \eta-\frac{\tan \psi}{3} \xi\right)
$$

is constant (as a function of $\xi$ ).

Proposition 2.23. Let $f:(\xi, \psi, \eta) \mapsto(\Xi, \Psi, H)$ be a minimiser satisfying the assumptions of Theorem 1.5. Then there exist continuous functions $G$ and $H_{0}$ such that

$$
\begin{aligned}
H(\xi, \psi, \eta) & =H_{0}(0, \eta)-G(0, \eta) \xi \\
& =H_{0}\left(\frac{\tan \psi}{3}, \eta+\frac{\tan \psi}{3} \xi\right)-G\left(\frac{\tan \psi}{3}, \eta+\frac{\tan \psi}{3} \xi\right) \xi
\end{aligned}
$$

for all $\xi \in(2 \log a, 2 \log b), \psi \in(-\pi / 2, \pi / 2)$ and $\eta \in \mathbb{R}$. 
Proof. The proposition is a consequence of the fact that $f$ sends radials to radials, and spherical arcs to spherical arcs. Radials in logarithmic coordinates are of the form

$$
s \mapsto(\xi(s), \psi(s), \eta(s))=\left(s, \psi, \eta-\frac{\tan \psi}{3} s\right) .
$$

Since $f$ maps radials to radials by scaling the Korányi gauge with power $k$, we must have for all $(\xi, \psi, \eta)$ that

$$
\left\{\begin{aligned}
H\left(s, \psi, \eta-\frac{\tan \psi}{3} s\right) & =H_{0}\left(\frac{\tan \psi}{3}, \eta\right)-\frac{\tan \Psi\left(s, \psi, \eta-\frac{\tan \psi}{3} s\right)}{3} k s \\
& =H_{0}\left(\frac{\tan \psi}{3}, \eta\right)-G\left(\frac{\tan \psi}{3}, \eta\right) s
\end{aligned}\right.
$$

for continuous functions $H_{0}$ and $G$. Inserting $\psi=0$, we find

$$
H(\xi, 0, \eta)=H_{0}(0, \eta)-G(0, \eta) \xi .
$$

Since $H$ is independent of $\psi$, we obtain that

$$
H(\xi, \psi, \eta)=H_{0}(0, \eta)-G(0, \eta) \xi
$$

for arbitrary $\psi$. At the same time, we find from the above formula for $H(s, \psi, \eta-$ $\left.\frac{\tan \psi}{3} s\right)$ that

$$
H(\xi, \psi, \eta)=H_{0}\left(\frac{\tan \psi}{3}, \eta+\frac{\tan \psi}{3} \xi\right)-G\left(\frac{\tan \psi}{3}, \eta+\frac{\tan \psi}{3} \xi\right) \xi,
$$

as claimed.

We use the functional equation (2.18) to derive more information on $H$. It is convenient to rewrite it by setting $u=\frac{\tan \psi}{3}$ and replacing $\eta$ in (2.18) with $\eta-u \xi$. We start with an abstract result.

Proposition 2.24. Let $G$ and $H_{0}$ be continuous functions on $\mathbb{R} \times \mathbb{R}$ that satisfy the functional equation

$$
H_{0}(0, \eta-u \xi)-G(0, \eta-u \xi) \xi=H_{0}(u, \eta)-G(u, \eta) \xi
$$

for all $\xi \in(\alpha, \beta), u, \eta \in \mathbb{R}$. Then there exist constants $c$ and $d$ such that

$$
G(u, \eta)=c u \quad \text { and } \quad H_{0}(u, \eta)=c \eta+d .
$$

Proof. Fix $u \neq 0$. By replacing in (2.20) the variable $\xi$ by $\xi+\frac{t}{u}$, and $\eta$ by $\eta+t$ for small $t$, and subtracting the resulting equation from (2.20), we find

$G(0, \eta-u \xi)-G(u, \eta+t)=u\left(\xi \frac{G(u, \eta+t)-G(u, \eta)}{t}-\frac{H_{0}(u, \eta+t)-H_{0}(u, \eta)}{t}\right)$.

Here we have to make sure that $t$ is small enough so that $\xi+\frac{t}{u} \in(\alpha, \beta)$. For any choice of $\xi \in(\alpha, \beta)$ there exists such an interval of admissible $t$ 's around 0 , but its length depends on $u$ and $\xi$. This does not cause problems since we will now take the limit $t \rightarrow 0$ in (2.21). Since the limit on the left-hand side exists by continuity, the limit on the right must also exist. Moreover, the factor $\xi$ ensures that the limit exists for both sums separately. Letting $t \rightarrow 0$ in (2.21), we conclude that the partial derivatives $G_{\eta}(u, \eta)$ and $H_{0 \eta}(u, \eta)$ exist for all $u \in \mathbb{R} \backslash\{0\}$ and all $\eta \in \mathbb{R}$ with

$$
G(0, \eta-u \xi)=u G_{\eta}(u, \eta) \xi-u H_{0 \eta}(u, \eta)+G(u, \eta)
$$

for all $\xi \in(\alpha, \beta), u \in \mathbb{R} \backslash\{0\}$ and $\eta \in \mathbb{R}$. Substituting the formula for $G(0, \eta-u \xi)$ in (2.20), we find in addition that

$$
H_{0}(0, \eta-u \xi)=u G_{\eta}(u, \eta) \xi^{2}-u H_{0 \eta}(u, \eta) \xi+H_{0}(u, \eta) .
$$


The idea is now to keep $u$ and $\eta$ fixed, and consider the above functions as functions of $x=\eta-u \xi$. It is easy to see that $x \mapsto G(0, x)$ and $x \mapsto H_{0}(0, x)$ are polynomials of degree 1 and 2, respectively, with coefficients that depend a priori on $u$ and $\eta$. The domain of $G(0, x)$ and $H_{0}(0, x)$, which is the interval $I_{u, \eta}=$ $\eta-u \cdot(\alpha, \beta)$, depends on $u$ and $\eta$, but for $(u, \eta)$ in a small enough neighborhood of a given $\left(u_{0}, \eta_{0}\right)$, the intersection $\bigcap I_{u, \eta}$ of the corresponding domains contains a non-empty interval $I$. We consider the functions $G(0, x)$ and $H_{0}(0, x)$ restricted to $I$ and conclude that the coefficients of the polynomials must be constant. Writing out the polynomials explicitly as

$$
G(0, x)=-G_{\eta}(u, \eta) x+\left(G_{\eta}(u, \eta) \eta-u H_{0 \eta}(u, \eta)+G(u, \eta)\right)
$$

and

$$
\begin{aligned}
H_{0}(0, x)= & \frac{1}{u} G_{\eta}(u, \eta) x^{2}+\left(-\frac{2 \eta}{u} G_{\eta}(u, \eta)+H_{0 \eta}(u, \eta)\right) x \\
& +\left(\frac{\eta^{2}}{u} G_{\eta}(u, \eta)-H_{0 \eta}(u, \eta) \eta+H_{0}(u, \eta)\right),
\end{aligned}
$$

we find that the coefficients must be constant in a neighborhood of $\left(u_{0}, \eta_{0}\right)$. From this we deduce that $G_{\eta}=0$ and $H_{0 \eta}=$ const. So finally, it follows that $G$ is a constant multiple of $u$ plus an additive constant, and $H_{0}$ a constant multiple of $\eta$ plus an additive constant. The constants depend a priori on the point $\left(u_{0}, \eta_{0}\right)$ in the neighborhood of which we did the computations, but a chaining argument then yields that they must be universal. Inserting $G(u, \eta)=c_{1} u+d_{1}$ and $H_{0}(u, \eta)=$ $c_{2} \eta+d_{2}$ in the functional equation (2.20), gives $c_{1}=c_{2}$ and $d_{1}=0$, as desired.

Corollary 2.25. Let $f:(\xi, \psi, \eta) \mapsto(\Xi, \Psi, H)$ be a minimiser satisfying the assumptions of Theorem 1.5. Then there exists a constant $\eta_{0}$ such that

$$
H=\eta+\eta_{0} \quad \text { and } \quad \Psi=\tan ^{-1}\left(\frac{\tan \psi}{k}\right) .
$$

Proof. By Proposition 2.23, minimisers satisfy the functional equation (2.18) for continuous functions $G$ and $H_{0}$. Since $f$ corresponds to a well-defined map on $S_{a, b}$, we have the periodicity condition in $\eta$, which forces us to take the constant $c$ in Proposition 2.24 equal to \pm 1 . The orientation preservation of $f$ excludes the possibility $c=-1$. This implies $G(u, \eta)=u$. Finally, from the definition of $G$ in (2.19) and with the substitution $u=\frac{\tan \psi}{3}$, we find

$$
\frac{\tan \psi}{3}=G\left(\frac{\tan \psi}{3}, \eta+\frac{\tan \psi}{3} s\right)=\frac{\tan \Psi(s, \psi, \eta)}{3} k,
$$

which concludes the proof.

2.5. Conclusion. We now collect the previously established results to conclude the proof of Theorem 1.5 .

Theorem 2.26. Let $\Gamma_{0}$ be the family of radial curves in $S_{a, b}$ and $\rho_{0}$ the associated extremal density. An orientation preserving quasiconformal map $f: S_{a, b} \rightarrow S_{a^{k}, b^{k}}$ which extends homeomorphically to the boundary and maps boundary components of $S_{a, b}$ to respective boundary components of $S_{a^{k}, b^{k}}$ satisfies

$$
k^{-3}=\mathfrak{M}\left(f_{k}, \rho_{0}\right)=\mathfrak{M}\left(f, \rho_{0}\right)
$$


if and only if

$$
\begin{aligned}
& \Xi(\xi, \psi, \eta)=k \cdot \xi, \\
& \Psi(\xi, \psi, \eta)=\tan ^{-1}\left(\frac{\tan \psi}{k}\right), \\
& \mathrm{H}(\xi, \psi, \eta)=\eta+\eta_{0} .
\end{aligned}
$$

Proof. The "if" part was shown in [4. The "only if" part follows from the results in this paper. First, Proposition 2.9 demonstrates that (2.22) is equivalent to

$$
M\left(f\left(\Gamma_{0}\right)\right)=\int_{S_{a, b}} K_{f}^{2} \rho_{0}^{4} \mathrm{~d} \mathcal{L}^{3},
$$

which allows us to apply Proposition 2.12 to deduce the minimal stretching property for the radials. Corollary 2.17 and Corollary 2.21 show further that a map $f$ that realises (2.22) has to preserve spheres (with $\Xi(\xi)=k \xi$ ), the $t$-axis and spherical arcs. Corollary 2.25 then yields the claimed formulae for $\Psi$ and $H$.

\section{Final Remarks and open questions}

The Beltrami differential $\mu_{f}=\frac{\bar{Z} f_{I}}{Z f_{I}}$ of the stretch map $f_{k}$ is

$$
\mu_{f_{k}}(\Phi(\xi, \psi, \eta))=-e^{3 i(\psi-\eta)} \frac{k^{2}-1}{k^{2}+1+\tan ^{2} \psi} .
$$

Its distortion function is

$$
K_{f_{k}}(\Phi(\xi, \psi, \eta))=K_{f_{k}}(\psi)=\frac{1+\tan ^{2} \psi}{k^{2}+\tan ^{2} \psi}
$$

with maximal distortion $K_{f_{k}}=1 / k^{2}$ if $k \in(0,1)$, and

$$
K_{f_{k}}(\Phi(\xi, \psi, \eta))=K_{f_{k}}(\psi)=\frac{k^{2}+\tan ^{2} \psi}{1+\tan ^{2} \psi}
$$

with maximal distortion $K_{f_{k}}=k^{2}$ if $k>1$.

There is an interesting analogy to the maps that realise equality in Belinskiu's inequality in the plane. Namely, for $0<k<1$, Belinskiıl's map $f(z)=|z|^{k} e^{i \Psi(\arg z)}$ (with appropriate assumptions on $\Psi$ ) has distortion

$$
K_{f}\left(r e^{i \varphi}\right)=\frac{\Psi^{\prime}(\varphi)}{k} .
$$

Our Heisenberg stretch satisfies

$$
K_{f_{k}}(\Phi(\xi, \psi, \eta))=\frac{\Psi^{\prime}(\psi)}{k} .
$$

The planar radial stretch map has a counterpart in $\mathbb{R}^{n}$. Belinskiǔ-type inequalities in higher dimensional Euclidean spaces have been established in [8, Theorem 2.9] and [11, Theorem 4.2], and sharpness can be shown by considering the radial stretch map in $\mathbb{R}^{n}$. We do not know whether the uniqueness question has been studied in this setting.

Question 3.1. Find all quasiconformal maps that realise equality in the Belinskiltype inequalities in $\mathbb{R}^{n}$. 
For minimisation problems related to other mean distortion functionals in the Euclidean setting, see [2, Theorem 14.1, References] and [1. In [3, Remark 22], we discuss further the example of two maps in Belinskilı's setting with the same weighted mean distortion, but a different maximal distortion. One of the two maps is the classical radial stretch in $\mathbb{C}$ for $k>1$, the other one is obtained by glueing a stretch with larger maximal distortion (defined on a subset of the annulus) to the identity. Theorem 1.5 shows that such a construction cannot work in the Heisenberg group. The heuristic behind this is the following: Since we consider the case $0<k<1$, the stretching in radial direction is prescribed for every minimiser, so the glueing should happen between different sectors of angular variables. Yet the contact condition is so strong that if the $\Xi$ component of a quasiconformal map is given, the $\Psi$ and $H$ components cannot be varied arbitrarily. We do not know the map of smallest maximal distortion in $\mathcal{F}$.

Question 3.2. Which maps in $\mathcal{F}$ minimise the $L^{\infty}$ norm of $K_{f}$ ?

In the planar Euclidean setting, Belinskiu [7] proved a second inequality similar to the one in Theorem 1.1, which reads as follows: For each $f \in \mathcal{F}$, one has

$$
k \cdot \log \left(\frac{b}{a}\right) \leq \frac{\int_{A_{a, b}} \frac{K_{f}(z)}{|z|^{2}} d \mathcal{L}^{2}(z)}{2 \pi} .
$$

If $0<k<1$, the inequality is strict. In the case $k>1$, equality is attained by the maps $f$ where $f(z)=z \Phi(|z|) e^{i \psi_{0}}$ with $r \mapsto r \Phi(r)$ being a $\mathcal{C}^{1}$ function $[a, b] \rightarrow\left[a^{k}, b^{k}\right]$ such that $\left|\Phi(r)+\Phi^{\prime}(r) r\right| \geq|\Phi(r)|, \Phi(r)+\Phi^{\prime}(r) r>0$ and $\Phi^{\prime}(r)>0$ on $[a, b]$, and $\psi_{0}$ fixed.

Question 3.3. Are the minimisers for the Heisenberg version of (3.1) unique up to composition with conformal maps?

\section{APPENDIX}

In this section, we provide several technical results that were used in the proof of Theorem 1.5

Proposition 3.4. Suppose that $\rho_{1}$ and $\rho_{2}$ are both extremal admissible densities for the 4-modulus of a curve family $\Gamma$ inside a domain $\Omega$. Then $\rho_{1}=\rho_{2}$ a.e. in $\Omega$.

Proof. We have

$$
\operatorname{Mod}(\Gamma)-\int_{\Omega}\left(\frac{\rho_{1}+\rho_{2}}{2}\right)^{4} \mathrm{~d} \mathcal{L}^{3} \leq 0
$$

or, equivalently,

$$
\int_{\Omega} \frac{\rho_{1}^{4}+\rho_{2}^{4}}{2}-\left(\frac{\rho_{1}+\rho_{2}}{2}\right)^{4} \mathrm{~d} \mathcal{L}^{3} \leq 0 .
$$

Yet, since $u \mapsto u^{4}$ is a strictly convex function, the integrand is non-negative, so by the above it must vanish almost everywhere and $\rho_{1}=\rho_{2}$ a.e. in $\Omega$.

Lemma 3.5. Assume that $\Phi: \Omega \rightarrow \mathbb{R}$ is a continuous function on a connected open set $\Omega=\underline{\Omega} \times \Omega_{n}$ in $\mathbb{R}^{n}$ such that for $\mathcal{L}^{n-1}$ almost every $\left(x_{1}, \ldots, x_{n-1}\right) \in \Omega$, the function $x_{n} \mapsto \Phi\left(x_{1}, \ldots, x_{n-1}, x_{n}\right)$ is constant. Then for all $\left(x_{1}, \ldots, x_{n-1}\right) \in \Omega$, the function $x_{n} \mapsto \Phi\left(x_{1}, \ldots, x_{n-1}, x_{n}\right)$ is constant. 
Proof. Assume towards a contradiction that there exists $\left(y_{1}, \ldots, y_{n-1}\right)$ such that

$$
x_{n} \mapsto \Phi\left(y_{1}, \ldots, y_{n-1}, x_{n}\right)
$$

is not constant. Thus there are distinct $u_{n}$ and $v_{n}$ so that $\Phi\left(y_{1}, \ldots, y_{n-1}, u_{n}\right) \neq$ $\Phi\left(y_{1}, \ldots, y_{n-1}, v_{n}\right)$. Consider then the continuous function $g: \underline{\Omega} \rightarrow \mathbb{R}$ defined by

$$
g\left(x_{1}, \ldots, x_{n-1}\right)=\Phi\left(x_{1}, \ldots, x_{n-1}, u_{n}\right)-\Phi\left(x_{1}, \ldots, x_{n-1}, v_{n}\right)
$$

Without loss of generality, $g\left(y_{1}, \ldots, y_{n-1}\right)>0$. Then also $g\left(x_{1}, \ldots, x_{n-1}\right)>0$ for $\left(x_{1}, \ldots, x_{n-1}\right)$ in a neighborhood of $\left(y_{1}, \ldots, y_{n-1}\right)$. Thus $x_{n} \mapsto \Phi\left(x_{1}, \ldots, x_{n}\right)$ is not constant for a positive measure set of points $\left(x_{1}, \ldots, x_{n-1}\right)$ in the neighborhood of $\left(y_{1}, \ldots, y_{n-1}\right)$, which is a contradiction.

\section{ACKNOWLEDGMENT}

We thank Irina Markina and Alexander Vasil'ev for discussions about the subject of Grötzsch-Belinskii inequalities.

\section{REFERENCES}

[1] K. Astala, T. Iwaniec, and G. Martin, Deformations of annuli with smallest mean distortion, Arch. Ration. Mech. Anal. 195 (2010), no. 3, 899-921, DOI 10.1007/s00205-009-0231-z. MR2591976 (2011c:30059)

[2] K. Astala, T. Iwaniec, G. J. Martin, and J. Onninen, Extremal mappings of finite distortion, Proc. London Math. Soc. (3) 91 (2005), no. 3, 655-702, DOI 10.1112/S0024611505015376. MR2180459(2006h:30016)

[3] Z. M. Balogh, K. Fässler, and I. D. Platis, Modulus of curve families and extremality of spiral-stretch maps, J. Anal. Math. 113 (2011), 265-291, DOI 10.1007/s11854-011-0007-x. MR2788359 (2012i:30045)

[4] Z. M. Balogh, K. Fässler, and I. D. Platis, Modulus method and radial stretch map in the Heisenberg group, Ann. Acad. Sci. Fenn. Math. 38 (2013), no. 1, 149-180, DOI 10.5186/aasfm.2013.3811. MR3076803

[5] Z. M. Balogh, P. Koskela, and S. Rogovin, Absolute continuity of quasiconformal mappings on curves, Geom. Funct. Anal. 17 (2007), no. 3, 645-664, DOI 10.1007/s00039-007-0607-x. MR2346270 (2009g:30023)

[6] Z. M. Balogh and J. T. Tyson, Polar coordinates in Carnot groups, Math. Z. 241 (2002), no. 4, 697-730, DOI 10.1007/s00209-002-0441-7. MR1942237 (2003k:22012)

[7] P. P. Belinskii, Obshchie svoistva kvazikonformnykh otobrazhenii (Russian), Izdat. "Nauka" Sibirsk. Otdel., Novosibirsk, 1974. MR0407275 (53 \#11054)

[8] C. J. Bishop, V. Ya. Gutlyanskiı̌, O. Martio, and M. Vuorinen, On conformal dilatation in space, Int. J. Math. Math. Sci. 22 (2003), 1397-1420, DOI 10.1155/S0161171203110034. MR.1980177 (2004c:30038)

[9] L. Capogna, D. Danielli, S. D. Pauls, and J. T. Tyson, An introduction to the Heisenberg group and the sub-Riemannian isoperimetric problem, Progress in Mathematics, vol. 259, Birkhäuser Verlag, Basel, 2007. MR2312336 (2009a:53053)

[10] N. S. Dairbekov, On mappings with bounded distortion on the Heisenberg group (Russian, with Russian summary), Sibirsk. Mat. Zh. 41 (2000), no. 1, 49-59, i, DOI 10.1007/BF02673994; English transl., Siberian Math. J. 41 (2000), no. 1, 40-47. MR.1756475 (2001f:30021)

[11] A. Golberg, Distortion of the Grötzsch-Belinskii type for generalized quasiconformal mappings, Georgian Math. J. 17 (2010), no. 2, 241-252. MR2676950(2011e:30066)

[12] A. Korányi and H. M. Reimann, Quasiconformal mappings on the Heisenberg group, Invent. Math. 80 (1985), no. 2, 309-338, DOI 10.1007/BF01388609. MR788413 (86m:32035)

[13] A. Korányi and H. M. Reimann, Foundations for the theory of quasiconformal mappings on the Heisenberg group, Adv. Math. 111 (1995), no. 1, 1-87, DOI 10.1006/aima.1995.1017. MR 1317384 (96c:30021) 
[14] A. Korányi and H. M. Reimann, Horizontal normal vectors and conformal capacity of spherical rings in the Heisenberg group (English, with French summary), Bull. Sci. Math. (2) 111 (1987), no. 1, 3-21. MR886958 (88e:22013)

[15] G. D. Mostow, Strong rigidity of locally symmetric spaces, Princeton University Press, Princeton, NJ; University of Tokyo Press, Tokyo, 1973. Annals of Mathematics Studies, No. 78. MR.0385004 (52 \#5874)

[16] P. Pansu, Métriques de Carnot-Carathéodory et quasiisométries des espaces symétriques de rang un (French, with English summary), Ann. of Math. (2) 129 (1989), no. 1, 1-60, DOI 10.2307/1971484. MR.979599 (90e:53058)

[17] I. D. Platis, The geometry of complex hyperbolic packs, Math. Proc. Cambridge Philos. Soc. 147 (2009), no. 1, 205-234, DOI 10.1017/S0305004109002333. MR2507316 (2010j:57029)

[18] J. Väisälä, Lectures on n-dimensional quasiconformal mappings, Lecture Notes in Mathematics, Vol. 229, Springer-Verlag, Berlin-New York, 1971. MR0454009 (56 \#12260)

Mathematisches Institut, Sidlerstrasse 5, CH-3012 Bern, Switzerland

E-mail address: zoltan.balogh@math.unibe.ch

Mathematisches Institut, Sidlerstrasse 5, CH-3012 Bern, Switzerland

E-mail address: katrin.faessler@math.unibe.ch

Department of Mathematics and Applied Mathematics, University of Crete, UniverSity Campus, GR-70013 Heraklion Crete, Greece

E-mail address: jplatis@math.uoc.gr 